\title{
Wie beeinflussen Arbeitsanreizerwartungen die sozialpolitischen Positionen der deutschen Arbeitgeberverbände?
}

\begin{abstract}
DE
Staatliche Sozialleistungen können, je nach Gestaltung des Leistungszugangs, Arbeitsanreize schwächen oder stärken. Eine Schwächung ergibt sich, wenn Leistungsbezug als Alternative zu Erwerbstätigkeit in Frage kommt; eine Stärkung wenn Leistungen an Verpflichtungen zur Arbeitssuche und Arbeitsaufnahme gekoppelt sind. Aus diesen Gründen ist die Gestaltung von Arbeitsanreizen durch Sozialpolitik für Arbeitgeber relevant. Dieser Beitrag untersucht durch historische Fallstudien von sechs sozialpolitischen Reformen welche Rolle die Spitzenverbände der Arbeitgeber Arbeitsanreizen in der Sozialpolitik beimaßen, sowie wie sich diese Einschätzungen im Zeitverlauf veränderten. Der Beitrag zeigt, dass die Verbände eine Reduktion des Arbeitskräfteangebots durch Sozialpolitik, je nach Arbeitsmarktlage, entweder negativ oder positiv bewerteten. Dort wo die Verbände eine Schwächung von Arbeitsanreizen als problematisch wahrnahmen, variierte die Intensität dieser Befürchtungen mit der Arbeitsmarktnähe des jeweiligen Programms.
\end{abstract}

\begin{abstract}
EN
Public social benefits can, depending on their design, either reduce or strengthen work incentives. Benefits that provide an alternative to earned income weaken work incentives, benefits that are linked to requirements to seek and accept work strengthen them. On the basis of six historical case studies, this paper analyses how the peak federations of German employers perceived the impact of public social programs on work incentives and how these perceptions changed over time. The paper shows how employers' assessments of the importance of work incentives changed over time: They perceived the negative work incentives created by social programs either as positive or as negative, depending on labor market conditions.
\end{abstract}

Schlüsselbegriffe: Unternehmen, Arbeitsanreize, Arbeitgeberverbände, Arbeitslosenversicherung, Frühverrentung.

\section{Kurzbiografie}


Dr. Thomas Paster ist Politikwissenschaftler und Visiting Fellow am Danish Center for Welfare Studies an der University of Southern Denmark. Sein derzeitiger Forschungsschwerpunkt liegt auf politischen Maßnahmen zur Begrenzung von Steuervermeidung durch multinationale Unternehmen. Er forschte zu Unternehmensinteressen in der Arbeitsmarkt- und Sozialpolitik und ist der Autor des Buchs "Containing Social Reforms: The Role of Business in the Development of the Welfare State and Labor Markets in Germany" (Routlege, 2012). Web: www.thomaspaster.com

Kontakt: Dr. Thomas Paster, Danish Center for Welfare Studies, University of Southern Denmark, Campusvej 55, 5230 Odense, Dänemark, Thomas.Paster at EUI.eu 


\section{Wie beeinflussen Arbeitsanreizerwartungen die sozialpolitischen Positionen der deutschen Arbeitgeberverbände?}

1. Einleitung ${ }^{1}$

Staatliche Sozialleistungen können Arbeitsanreize reduzieren in dem sie Bürgern eine Alternative zum Lebensunterhalt durch Markteinkommen eröffnen. Eine großzügige Ausgestaltung des Zugang zu Sozialleistungen kann das Arbeitskräfteangebot reduzieren indem die Bereitschaft von nicht-erwerbstätigen Personen Arbeit anzunehmen geschwächt wird (siehe z.B. Esping-Andersen 1990: 22). Sozialleistungen können jedoch auch Arbeitsanreize fördern, in dem Leistungsansprüche an Verpflichtungen zur Arbeitssuche und Arbeitsaufnahme geknüpft werden. Die Ausgestaltung staatlicher Sozialpolitik hat daher Auswirkungen auf die Sicherstellung eines ausreichenden und qualifizierten Arbeitskräfteangebots für Unternehmen.

Negative Arbeitsanreize durch Sozialpolitik müssen jedoch nicht ungewollt sein, sondern können Teil einer politischen Strategie des Umgangs mit Arbeitslosigkeit sein. Die Reduktion des Arbeitskräfteangebots durch staatliche Sozialpolitik in Perioden hoher Arbeitslosigkeit kann eine politisch intendierte Maßnahme sein um den durch strukturelle Arbeitslosigkeit verursachten politischen Problemdruck zu reduzieren (siehe bspw. Mares 2004b; Ebbinghaus 2006; Kohli/Rein/Guillemard/van Gunsteren 1991).

Da Arbeitgeber von solchen Effekten unmittelbar betroffen sind, stellt sich die Frage wie diese die Arbeitsanreizeffekte von Sozialpolitik wahrnehmen und wie die politischen Vertreter der Arbeitgeberinteressen, in erster Linie die Arbeitgeberverbände, versuchen die Stärkung oder Schwächung von Arbeitsanreizen durch Sozialpolitik mitzugestalten und zu beeinflussen. Ist die Befürchtung negativer Arbeitsanreize ein wesentlicher Grund für eine eventuelle Ablehnung staatlicher Sozialpolitik durch die Arbeitgeberverbände? Betrachten die Arbeitgeberverbände staatliche Sozialpolitik als eine Gefährdung von Arbeitsanreizen? Falls ja, wie erfolgreich sind die Arbeitgeberverbände dabei arbeitsanreizreduzierende Regulierungen in der Sozialpolitik zu verhindern?

Dieser Beitrag untersucht durch einen diachronischen Vergleich von Reformprojekten in der Entwicklung des Sozialstaats in Deutschland, erstens wie die Spitzenverbände der Arbeitgeber die Auswirkungen sozialpolitischer Reformen auf den Erhalt von Arbeitsanreizen wahrnahmen, und zweitens, wie diese Wahrnehmungen deren Positionen zu diesen

\footnotetext{
${ }^{1}$ Für hilfreiche Hinweise danke ich Daniel Kinderman und den beiden anonymen Gutachtern bzw. Gutachterinnen. Die Arbeit an diesem Beitrag wurde durch das Horizon 2020 Forschungsprogramm der Europäischen Union unter der Marie Sklodowska-Curie Fördervereinbarung Nr. 705677 unterstützt.
} 
Reforminitiative beeinflussten. In welchen Fällen sahen sie eine Reduktion des Arbeitskräfteangebots als Gefahr, in welchen als Chance?

Der Beitrag untersucht dabei sechs Reformprojekte: Die Einführung der staatlichen Unfallversicherung (1884), die Einführung der staatlichen Krankenversicherung (1883), die Einführung der Alters- und Invalidenrenten (1889), die Einführung der staatlichen Arbeitslosenversicherung (1927), die Ausweitung der Möglichkeiten zur Frühverrentung in den 1980ern, und die Agenda 2010 Arbeitsmarktreformen (2003-2005). Diese Reformepisoden und Sozialprogramme wurden wegen ihrer zentralen Bedeutung für die Entwicklung des Sozialstaats in Deutschland ausgewählt, in dem Sinne das durch die ersten vier dieser Reformen die programmatischen Grundlagen für die spätere Entwicklung gelegt wurden. Mit der Inklusion der Frühverrentungspolitik und der Agenda 2010 Reformen wird die Analyse über die Entstehungsphase des modernen Sozialstaats hinaus auf Entwicklungen der letzten Jahrzehnte hin ausgedehnt.

Die Periode des NS-Regimes klammert de Beitrag aus, da der Arbeitsmarkt in dieser Zeit in beträchtlichem Maß auf Zwang und gewaltsamer Rekrutierung in den besetzten Gebieten basierte und daher die Thematik der Arbeitsanreize im Vergleich mit der liberaldemokratischen Variante des Kapitalismus sehr unterschiedlich zu analysieren und zu beurteilen wäre. ${ }^{2}$

Der Fokus der Analyse liegt auf den Positionen der jeweiligen Spitzenverbände der Arbeitgeber, da diese in der Sozialpolitik das tonangebende Sprachrohr der Unternehmen sind. Bei den untersuchten Verbänden handelt es sich dabei um den Centralverband deutscher Industrie (CVDI) im Kaiserreich, der Vereinigung deutscher Arbeitgeberverbände (VDA) in der Weimarer Republik, und der Bundesvereinigung deutscher Arbeitgeberverbände in der Bundesrepublik. Mitglieder des jeweiligen nationalen Spitzenverbands waren bzw. sind nationale Branchenverbände (heute „Bundesfachspitzenverbände“ genannt) und Landesverbände, wobei jedoch nicht alle existierenden Branchenverbände Mitglied im jeweiligen Spitzenverband sind bzw. waren.

Zudem entwickelte sich in Deutschland ab dem späten 19. Jahrhundert eine Zweiteilung in Industrieverbände und Arbeitgeberverbände, wobei die ersteren die wirtschaftspolitischen Interessen ihrer Mitglieder zu vertreten beanspruchen, die letzteren die

\footnotetext{
2 Die Studien der Unabhängigen Historikerkommission zum Reichsarbeitsministerium berücksichtigen auch die Arbeits- und Sozialpolitik des NS-Regimes und dokumentieren unter anderem auch die bekannte Rolle des RAMs bei der Organisation des Arbeitseinsatzes (Nützenadel 2017). Unbestritten ist dabei, dass die Organisation des Arbeitsmarktes in der NS-Regime stärker auf Zwang und Zuteilung beruhte, und nicht so sehr auf der Gestaltung von Arbeitsanreizen. Gegen Ende des Krieges waren etwa 6 Millionen zivile ausländische Beschäftigte als Zwangsarbeiter eingesetzt. Zur Arbeit eingesetzte Kriegsgefangene und Insassen der Konzentrationslager sind in dieser Zahl nicht enthalten. Arbeitsplatzwechsel konnte verboten und Beschäftigte gegen ihren Willen für Arbeiten von „staatswichtiger Bedeutung“ eingesetzt werden.
} 
sozial-und tarifpolitischen Interessen. Der CVDI, gegründet 1876 von den Verbänden der Schwerindustrie zum Zweck der Durchsetzung von Schutzzöllen, positionierte sich ab den späten 1870ern auch zu sozialpolitischen Themen, insbesondere zu den Bismarckschen Reformplänen (Kaelble 1967; Bueck 1905a). Der erste Arbeitgeber-Spitzenverband auf nationaler Ebene wurde 1904 gegründet (Bueck 1905b), aus dieser Organisation ging durch Zusammenschluss mit einer konkurrierenden Organisation die 1913 gegründete VDA hervor, welche in der Weimarer Republik das primäre Sprachrohr für Arbeitgeberinteressen in der Sozialpolitik wurde. Die BDA wurde 1949 gegründet, nachdem von den Alliierten das Organisationsverbot für Arbeitgeber auf Interzonenebene aufgehoben wurde.

Die Branchenverbände waren und sind primär in der Tarifpolitik tätig und beeinflussen die sozialpolitische Positionsbildung des nationalen Spitzenverbands durch interne Abstimmung, agieren jedoch in den öffentlichen Debatten um sozialpolitische Reformen zurückhaltend, da dieser Kompetenzbereich beim nationalen Spitzenverband liegt. Aus diesem Grund untersucht der Beitrag die Positionen der Branchenverbände nicht systematisch. Unterschiedliche Branchen und Arten von Unternehmen können jedoch unterschiedliche Interessen in der Sozialpolitik haben. Die Positionen der Spitzenverbände werden bzw. wurden nicht zwingend von allen Mitgliedsverbänden oder Mitgliedsunternehmen geteilt. Wo solche Differenzen beobachtbar sind, werden diese im Beitrag diskutiert.

Um die Argumente und Positionen der Verbände zu dokumentieren, verwendet der Beitrag historische Quellen, in erster Linie Publikationen und Zeitschriften der Arbeitgeberverbände, die Jahresberichte der Verbände, und interne Positionspapiere.

\subsection{Thesen und Periodisierung}

Die Hauptthese des Beitrag ist, dass die Spitzenverbände der Arbeitgeber negative Arbeitsanreizeffekte von Sozialprogrammen je nach Arbeitsmarktlage und je nach Programm unterschiedlich beurteilten. Diese unterschiedliche Beurteilung analysiert der Beitrag auf Basis von zwei Vergleichsdimensionen. Erstens, werden die Positionen diachron, also zwischen historischen Perioden, verglichen. Zweitens, werden, soweit möglich, Verbandspositionen zu arbeitsmarktnahen und arbeitsmarktfernen Programmen verglichen, wobei bei arbeitsmarktnahen Programmen eine höhere Relevanz von Arbeitsanreizeffekten vermutet wird. Arbeitsmarktnahe Programme sind solche, welche arbeitsfähigen Personen zugute kommen, wie der Arbeitslosenversicherung und der Erwerbslosenfürsorge. Arbeitsmarktferne Programme sind solche welche Personen zugute kommen welche dem 
Arbeitsmarkt temporär oder dauerhaft nicht zur Verfügung stehen, wie Kranke, Invalide, oder ältere Menschen.

Für den diachronen Vergleich werden die Reformen in fünf Perioden unterteilt. In der ersten Phase, der Phase des Einführung staatlicher Sozialversicherungsprogramme im Kaiserreich befürchteten die Verbände zwar negative Arbeitsanreize, da die eingeführten Programme; das heißt, die Unfallversicherung, die Krankenversicherung, und die Alters- und Invaliditätsrenten; jedoch arbeitsmarktfern waren, waren diese Befürchtungen bei der Gesamtbeurteilung dieser Reformen für den Centralverband nicht entscheidend und führten zu keiner grundsätzlichen Ablehnung.

In der zweiten Phase, der Weimarer Republik, prägten Befürchtungen von negativen Arbeitsanreizen die sozialpolitischen Positionen der Verbände stärker. Am stärksten lehnten die Arbeitgeberverbände die Einführung der Arbeitslosenversicherung ab, da sie, wie der Beitrag zeigt, eine Schwächung von Arbeitsanreizen befürchteten. Bei der Einführung der Krankenversicherung, der Unfallversicherung, sowie der Alters- und Invalidenrenten hingegen leisteten die Arbeitgeber deutlich weniger Widerstand, da diese Programme nur Personen zugute kommen welche dem Arbeitsmarkt, temporär oder dauerhaft, nicht zur Verfügung stehen.

In der dritten Phase, der sozialpolitischen Expansionsphase der Nachkriegsjahrzehnte, blieb der Erhalt von Arbeitsanreizen für die Arbeitgeberverbände eine relevante Zielsetzung, diese übersetzte sich jedoch, im Unterschied zur Weimarer Republik, nicht in eine grundsätzliche Ablehnung von expansiven Reformen, sondern in Bestrebungen Sozialprogramme arbeitsanreizkompatibel zu gestalten. In der vierten Phase, ab den 1970ern, begannen die Verbände im Kontext von Überbeschäftigung und Umstrukturierungen in der Industrie eine Reduktion des Arbeitskräfteangebots durch Sozialpolitik als wünschenswert zu betrachten. Die Verbände begrüßten nun negative Arbeitsanreizeffekte. In der fünften Phase, ab de1990ern, betonen die Verbände hingegen wieder verstärkt die Notwendigkeit zur Stärkung und Erhalt von Arbeitsanreizen durch Sozialpolitik.

Diese Veränderungen in der Einschätzung von Arbeitsanreizeffekten durch die Spitzenverbände der Arbeitgeber korrelieren mit Änderungen in der Arbeitsmarktlage aber auch mit positiven und negativen Erfahrungen mit Sozialpolitik in der jeweils vorhergehenden Periode. Die untersuchten Reformen fanden in unterschiedlichen politischen, kulturellen, und institutionellen Kontexten statt, welche die Einschätzung der Arbeitsanreizeffekten durch die Arbeitgeberverbände möglicherweise beeinflussten, deren kausale Bedeutung jedoch wegen der begrenzen Fallzahl (6) in diesem Aufsatz nicht 
abschließend beurteilt werden kann. In de Phase des Aufbaus staatlicher Sozialpolitik bis in die Weimarer Republik, während der Erfahrungen mit Arbeitsanreizeffekten von bestehenden Sozialprogrammen noch wenig bestanden, war die ablehnende Haltung der Verbände eine grundsätzliche und variierte lediglich mit der Arbeitsmarktbähe des jeweiligen Programms. So war die Arbeitslosenversicherung jener Zweig der Sozialversicherung welchen die Arbeitgeberverbände am stärksten ablehnten, wobei, wie der Beitrag zeigt, die Befürchtung negativer Arbeitsanreize das primäre Motiv war, und erst in zweiter Linie die Befürchtung höherer Arbeitskosten.

In der Phase des Strukturwandels in der Industrie in den 1970ern und 1980ern, erkannten Arbeitgeber schließlich negative Arbeitsanreize durch Sozialpolitik als für die gesellschaftliche Gestaltung von Personalabbau zweckmäßiges Mittel. Der Beitrag analysiert diesen Wandel anhand der Vorruhestandspolitik. Seit den 1990ern treten die Arbeitgeberverbände im Kontext gestiegener Sozialausgaben und eines prognostizierten Arbeitskräftemangels, wieder für eine Stärkung von Arbeitsanreizen in der Sozialpolitik ein. Im Unterschied zur Anfangsphase staatlicher Sozialpolitik argumentieren die Verbände jedoch heute nicht grundsätzlich gegen Sozialpolitik, sondern fokussieren sich darauf die Ausgestaltung von Leistungsansprüchen arbeitsanreizkompatibel zu gestalten, beispielsweise durch den Abbau versicherungsfremder Leistungen.

Der Beitrag zeigt daher durch Betrachtung eines langen Zeitraums, dass die Interessensinterpretation der Arbeitgeber einem historischen Wandel unterlag. Besonders die Arbeitsmarktlage und bestehende Erfahrungshorizonte beeinflussen wie die Arbeitgeberverbände die Arbeitsanreizeffekte von Sozialpolitik beurteilen. Sie betrachten diese daher manchmal als problematisch, manchmal als wünschenswert.

\subsection{Relevanz der Ergebnisse für die Forschung zu Arbeitgeberpräferenzen}

Zum Einfluss von Arbeitgeberverbänden auf sozialpolitische Reformen besteht eine umfangreiche Forschungsliteratur. Diese Literatur untersucht welche Haltungen unterschiedliche Verbände zu sozialpolitischen Themen einnehmen und welche Präferenzen und Interessensdeutungen diesen Haltungen zugrunde liegen (siehe bspw. Bührer 2010; Busemeyer 2011; Callaghan 2011; Ebbinghaus 2001, 2010; Eichhorst/Weishaupt 2013; Emmenegger/Marx 2011; Höpner/Waclawczyk 2012; Kinderman 2016; Mares 2004b; Münnich 2010; Pancaldi 2011; Paster 2017; Schroeder 2006; Streeck 2016; Trampusch 2004). ${ }^{3}$ Die in diesem Forschungsfeld betrachteten Erklärungsfaktoren für unterschiedliche

${ }^{3}$ Einen detaillierteren Literaturüberblick dazu bietet 
Haltungen lassen sich in zwei Gruppen einteilen: solche welche eine positive Haltung gegenüber staatliche Sozialpolitik erklären können, und solche eine negative Haltung erklären können. Im Hinblick auf die Funktionsweise des Arbeitsmarktes werden in diesem Forschungsfeld zwei Faktoren als relevant betrachtet: Ausbildungsanreize und Arbeitsanreize. Die Erwartung von Ausbildungsanreizen wird dabei häufig als ursächlich für eine positive Haltung von Arbeitgeberverbänden gegenüber Sozialpolitik angesehen; die Erwartung von negativen Arbeitsanreizen hingegen als ursächlich für eine ablehnende Haltung (siehe Paster 2019).

Ähnlich wie einige bestehende Studien (Brosig 2011; Bridgen 2016; Korpi 2006; Paster 2013, 2020), betont dieser Beitrag die Rolle von Arbeitsanreizen. Jedoch geht dieser Beitrag über den Forschungsstand hinaus indem er für Deutschland den Wandel der Haltungen der Verbände zu Arbeitsanreizen untersucht. Dadurch kann der Beitrag zeigen, dass Erwartungen negativer Arbeitsanreize nicht $\mathrm{zu}$ einer konstanten Ablehnung von Sozialpolitik durch die Arbeitgeberverbände, sondern je nach Arbeitsmarktlage und abhängig von bestehenden Erfahrungen mit staatlicher Sozialpolitik, entweder zu einer ablehnenden oder befürwortenden Haltung führten. Des weiteren zeigt der Beitrag, dass die Bedeutung des Themas Arbeitsanreize für die Arbeitgeberverbände je nach Arbeitsmarktnähe des jeweiligen Programms variierte. Der Rest dieses Abschnitts diskutiert die beiden in der Literatur angenommenen Motivationsfaktoren, Ausbildungsanreize und Arbeitsanreize, und evaluiert diese in Bezug auf die Ergebnisse dieses Beitrags.

Die Kernthese dieses Beitrags unterscheidet sich von Argumenten in der politökonomischen Forschungsliteratur welche primär die Rolle von Ausbildungsanreizen für die sozialpolitische Präferenzbildung der Arbeitgeberverbände in den Blick nimmt. Damit sind theoretische Modelle gemeint, welche davon ausgehen, dass staatliche Sozialleistungen Anreize für Investitionen in berufs-oder branchen-spezifische Qualifikationen schaffen und aus diesem Grund von jenen Gruppen von Unternehmen welche diese Typen von Qualifikationen benötigen befürwortet werden (siehe bspw. Estévez-Abe/Iversen/Soskice 2001: 180-181; Iversen 2005).

Diese theoretische Perspektive ist vom Ansatz der Spielarten des Kapitalismus (Hall/Soskice 2001) inspiriert und geht davon aus, dass Arbeitnehmer es vorziehen in Qualifikationen zu investieren welche zwischen Firmen und Branchen transferierbar sind. Sozialpolitische Maßnahmen, darunter insbesondere eine staatliche Arbeitslosenversicherung, reduzieren das Risiko einer Nichtverwertbarkeit von Ausbildungsinvestitionen durch Veränderungen der Arbeitskräftenachfrage. Es wird daher in dieser theoretischen Perspektive 
erwartet, dass staatliche Sozialpolitik die Bereitschaft von Arbeitnehmern in nichttransferierbare Qualifikationen zu investieren erhöht.

Aus diesen theoretischen Überlegungen lässt sich die Hypothese generieren, dass jene Unternehmen welche auf nicht-transferierbare Qualifikationen angewiesen sind, staatlichen Sozialleistungen positiver gegenüberstehen werden. Diese These vertritt beispielsweise Isabela Mares in Studien zu sozialpolitischen Reformen in Deutschland und Frankreich (Mares 2003, 2004a: 758). Die in diesem Beitrag präsentierten Forschungsergebnisse stützen diese These jedoch nicht und zeigen, dass die Spitzenverbände der Arbeitgeber bei ihrer Beurteilung sozialpolitischer Maßnahmen Arbeitsanreizen eine wesentlich größere Rolle beimessen als Ausbildungsanreizen.

Die These einer zentralen Relevanz von Erwartungen von negativen Arbeitsanreizen für die sozialpolitischen Haltungen der Arbeitgeberverbände hat ihre Wurzel im Machtresourcenansatz, welcher Sozialleistungen als Stärkung der relativen Machtposition von Arbeitnehmern im Verhältnis zu Arbeitgebern interpretiert. Diese These brachte beispielsweise Esping-Andersen in seinen Drei Welten des Wohlfahrtskapitalismus zum Ausdruck:

De-Kommodifizierung stärkt den Arbeiter und schwächt die absolute Autorität des Arbeitgebers. Eben deshalb haben sich letztere immer gegen die DeKommodifizierung gesträubt (Esping-Andersen 1990: 22).

Die Ergebnisse dieses Beitrags legen nahe, dass in Deutschland dieses Präferenzmodell auf die Phase des Aufbaus staatlicher Sozialprogramme im Kaiserreich und in der Weimarer Republik zutrifft.

Die Ergebnisse zeigen jedoch auch, dass dieses Modell auf die Phase der Vorruhestandspolitik in den 1970er und 1980er nicht anwendbar ist, da in dieser Phase die Spitzenverbände der pazifizierenden Wirkung von Sozialleistungen eine größere Bedeutung als der arbeitsanreizreduzierenden Wirkung beimaßen. Die These einer grundsätzlichen und konstanten Ablehnung staatlicher Sozialpolitik durch Arbeitgeberverbände erscheint daher im Lichte dieser Ergebnisse als zu statisch, da die Arbeitsmarktnähe sozialpolitischer Programme und die Arbeitsmarktlage empirisch variieren. Allerdings erklären nicht erwartete Ausbildungsanreize positive Haltungen der Verbände zu Sozialpolitik, sondern die Erwartung der Möglichkeit eines konfliktarmen Personalabbaus in Phasen der Überbeschäftigung.

Dieser Beitrag geht daher empirisch und theoretisch dadurch über den Forschungsstand hinaus, indem er zeigt wie die Spitzenverbände der Arbeitgeber die Rolle von negativen Arbeitsanreizen durch staatliche Sozialpolitik in unterschiedlichen Kontexten 
unterschiedlich beurteilten und dadurch $\mathrm{zu}$ unterschiedlichen Positionen kamen. Die Präferenzmodellierung des Machtressourcenansatzes erweist sich für die Phase der Einführung und des Ausbaus der staatlichen Sozialprogramme bis in die 1960er als gültig, für die Phase ab der 1970er jedoch als nicht anwendbar. Für die auf Ausbildungsanreize basierende Präferenzmodellierung findet der Beitrag keine Bestätigung.

Der Beitrag weißt daher auf die Bedeutung von periodenspezifischen Präferenzbildungsmustern hin. Die Beantwortung der in der Literatur diskutierten Frage ob Fälle in denen Arbeitgeberverbände sozialpolitischen Reformen zustimmten durch genuine oder durch strategische Präferenzen erklärt werden können muss diese periodenspezifische Beurteilung der Wirkungen von Sozialprogrammen berücksichtigten. So war die Zustimmung der Arbeitgeberverbände zur Einführung der staatlichen Arbeitslosenversicherung strategischer Natur, die Unterstützung der Vorruhestandspolitik jedoch beruhte auf einer genuinen Interessenskonstruktion, wenn gleich diese Konstruktion sich als temporär herausstellte. Kurz gefasst sind also die Gründe für die Akzeptanz sozialpolitischer Reformen durch die Spitzenverbände sowohl perioden- als auch programmspezifisch, wobei die Verbände die Bedeutung von Arbeitsanreizen je nach Arbeitsmarktnähe des jeweiligen Programms und je nach Arbeitsmarktlage unterschiedlich beurteilten. Ein genuines Interesse an staatlicher Sozialpolitik sahen die Spitzenverbände nur in Perioden der Überbeschäftigung, jedoch nicht im Zusammenhang mit Ausbildungsanreizen.

Die Präsentation der Ergebnisse in diesem Beitrag folgt einer im groben Zügen chronologischen Ordnung: Der Beitrag beginnt mit einer Analyse der Arbeitgeberhaltungen zur Einführung einer staatlichen Unfallversicherung Anfang der 1880er, gefolgt von der Einführung der staatlichen Rentenversicherung und Krankenversicherung in den 1880ern, der Einführung der staatlichen Arbeitslosenversicherung in den 1920ern, die sozialpolitischen Reformen der unmittelbaren Nachkriegszeit und zuletzt der Arbeitsmarktreformen in den 2000ern. Ein abschließender Abschnitt fasst die Ergebnisse zusammen.

\section{Die Einführung der staatlichen Unfallversicherung}

Die Einführung der Unfallversicherung war das erste der drei unter Bismarck eingeführten Programme, Krankenversicherung und Rentenversicherung die beiden anderen. Die Einführung der Unfallversicherung löste die Haftpflichtgesetzgebung von $1871 \mathrm{ab}$, welche Arbeitgeber zur Entschädigung von Arbeitern bei Arbeitsunfällen verpflichtete, sofern das Verschulden beim Arbeitgeber lag, wobei gerichtliche Auseinandersetzungen über die Schuldfrage häufig vorkamen (Breger 1994: 24-28). 
Vertreter der Industrie spielten bei der Einführung eine wichtige Rolle, insbesondere die Schwerindustrie, welche zu diesem Zeitpunkt bereits wesentlich stärker in Verbänden organisiert war als andere Branchen. Da Hauptinteresse der Industrie galt der Überwindung der Haftpflicht bei Arbeitsunfällen durch Übertragung der Entschädigungsfunktion auf ein staatliches Programm und dadurch die Entschärfung des Konfliktpotentials in den Betrieben im Kontext der entstehenden Gewerkschaften.

Die wichtigste Initiative der Arbeitgeberseite im Kontext der Unfallversicherung war eine Konferenz von Industrievertretern in Bochum am 2. Und 3. November 1880, welche von Louise Baare, Direkter des Stahlunternehmens Bochumer Verein und Präsident der Handelskammer Bochum, organsiert wurde. Baare hatte bereits am 30. April 1880 beim Preussischen Wirtschaftsministerium für die Einführung einer staatlichen Unfallversicherung interveniert (Breger 1994: 26-27). Die Gruppe um Louise Baare, bestehend aus etwa 30 Vertretern der Schwerindustrie und anderer Zweige der Großindustrie, entwarf einen Vorschlag für eine staatliche Unfallversicherung, gedacht als Alternative zu einer Verschärfung der Arbeitgeber-Haftpflicht und des Arbeiterschutzes, Maßnahmen welche von den Gewerkschaften gefordert wurden(Baare 1880). Der Entwurf der Baare Gruppe sah eine verpflichtende Mitgliedschaft für alle Arbeitnehmer in allen Branchen in einer staatlich organisierten Versicherung vor, welche durch Beiträge von Arbeitgebern (50 Prozent), Arbeitnehmern (25 Prozent) und Subventionen de Kommunen (25 Prozent) zu finanzieren sei und von einem Reichsversicherungsamt zu verwalten sei.

Der Vorschlag genoss offenbar innerhalb der Industrie große Unterstützung. Der Centralverband deutscher Industrieller (CVI), der größte Dachverband der Wirtschaftsverbände zu dieser Zeit, sprach sich für den Vorschlage der Gruppe von Louise Baare aus (Breger 1994: 26). Eine Umfrage des Königlich-Preussischen Statistischen Bureaus unter den regionalen Handels-und Gewerbekammern zeigt jedoch ein differenzierteres Bild. Gemäß dieser Umfrage, gaben 53 Prozent der befragten Kammern an, den Regierungsentwurf von 1881 für eine Unfallversicherung als eine Verbesserung im Vergleich zum Haftpflichtgesetz von $8171 \mathrm{zu}$ sehen. 26 Prozent bevorzugten eine Reform der Haftpflichtgesetzgebung gegenüber einer Unfallversicherung(Francke 1881: 397-398).

Befürchtungen einer Erosion von Arbeitsanreizen durch eine Unfallversicherung spielten eine untergeordnete Rolle. Aus dem Protokoll der Bochumer Konferenz geht hervor, dass deren Teilnehmer Wert darauf legten die Leistungshöhen gering zu halten, konkret sollten diese maximal zwei Drittel des Nettolohns ausmachen um Anreize für Leistungsbetrug zu reduzieren. Ein Rechtsanspruch auf Leistungen sollte nicht bestehen, und eine Ausdehnung 
des Programms zu einer Alters- und Krankenversicherung sei zu unterlassen (Baare 1880). Zusammenfassend lässt sich also sagen, das die involvierten Industrievertreter zwar die Möglichkeit einer Erosion von Arbeitsanreizen durch Leistungsbetrug ins Auge gefasst hatten, diese Befürchtungen jedoch im Fall der Unfallversicherung für deren Gesamteinschätzung des Programms nicht ausschlaggebend waren. Da sich Arbeitsunfälle leicht dokumentieren lassen, erschien den Arbeitgebern in diesem Fall die Gefahr von negativen Arbeitsanreizen offenbar als gering,

\section{Die Einführung der Rentenversicherung und der Krankenversicherung}

Die Einführung der staatlichen Altersrenten -und Invalidenversicherung, sowie der staatlichen Krankenversicherung waren die weiteren Säulen der Bismarck'schen Sozialreformen. Auch bei diesen Programmen war die Haltung der Industrieverbände zum Ausbau der sozialen Sicherung zwar nicht die eines Vorkämpfers, jedoch eine grundsätzlich akzeptierende und kooperierende. Die auf Seiten der Arbeitgeber einflussreichsten Verbände waren die Verbände der Schwerindustrie sowie der CVDI, welcher von der Schwerindustrie im Ruhrgebiet und der Textilindustrie in Süddeutschland dominiert wurde. Die verarbeitende Industrie war zu diesem Zeitpunkt noch nicht in einem nationalen Spitzenverband organisiert und hatte wenig Einfluss auf den politischen Entscheidungsfindungsprozess (Ullmann 1979). Für die politisch einflussreiche Schwerindustrie war der Erhalt guter Beziehungen zur Regierung ein wichtiges Motiv für die kooperative Haltung beim Entwurf der staatlichen Sozialversicherung.

Welche Argumente brachten die Industrievertreter bei den Bismarck'schen Reformen ein? Neben dem Bestreben Kostensteigerungen durch die Ausgestaltung von Leistungsansprüchen in Grenzen zu halten (Bueck 1905a: 792), spielte bei der Krankenversicherung die Rolle der gewerkschaftsnahen freien Hilfskassen eine Rolle. Diese wurden von der Arbeitgeberseite abgelehnt und sollten nicht in die öffentliche Krankenversicherung integriert werden.

Befürchtungen einer Erosion der Arbeitsanreize spielten bei der Debatte um die Invaliditäts- und Altersrenten eine Rolle. Aus den Quellen gehen jedoch keine Hinweise hervor, dass Überlegungen zu Arbeitsanreizen bei der Einführung der Krankenversicherung eine Rolle gespielt hätten. Da diese ja Personengruppen zugute kommt, welche temporär nicht arbeitsfähig sind, hatten die Arbeitgeberverbände keine grundsätzlichen Einwände gegen die Einführung einer Krankenversicherung. Der CVDI gab an mit der Einführung einer Altersrente zu „sympathisieren“, jedoch sollte deren Einführung verzögert werden und 
Leistungen so niedrig wie möglich sein (Bueck 1905a: 324). Ähnliche Positionen vertraten auch der Verein Deutscher Eisen-und Stahl-Industrieller (VDESI) und der Langnam-Verein, welcher die Interessen der Schwerindustrie im Rheinland und in Westfalen vertrat (Breger 1982: 134-137).

Aus den Stellungnahmen der Industrieverbände gehen dabei vor allem zwei Argumente gegen Alters- und Invaliditätsrenten hervor. Erstens, argumentierten die Verbände mit Kostensteigerungen. Die Einführung der Unfall- und der Krankenversicherung hätten die Arbeitskosten erhöht und es sollte daher zunächst mit weiteren Reformen gewartet werden, wie Henry Axel Bueck, Geschäftsführer des CVDI von 1887 bis 1916, in seiner Verbandsbiographie schreibt (Bueck 1905a). Zweitens, würden Alters- und Invalidenrenten Möglichkeiten zum Leistungsbetrug für „Drückeberger“ schaffen. Insbesondere der Tatbestand der Arbeitsunfähigkeit sei schwer nachzuweisen (Breger 1982: 133-135). Bei der 11. Generalversammlung des Langnamvereins am 29. November 1881 meinte dessen Geschäftsführer, Henry Axel Bueck, welcher wie erwähnt später Geschäftsführer des CVDI wurde:

[W]enn von Staats wegen jedem Arbeiter von vornherein eine Pension für die Tage der Invalidität und Altersschwäche als ein von ihm in Anspruch zu nehmendes Recht zugesagt würde, so würde man das Gefühl der Selbstverantwortlichkeit vermindern, und das würde der größte Schaden sein, den wir unseren sozialen Verhältnissen zufügen könnten. .... Ich habe gesagt ... "Arm zu sein, muß niemals aufhören ein Unglück zu sein." Es mag dies sehr hart, sehr herzlos klingen aber entfernen Sie heute die Perspektive, welches Elend, Mangel und Not als die Folgen von Sorglosigkeit, Indolenz, Trägheit und Liederlichkeit hinstellt, so wird mit diesem Augenblick ein großer Prozentsatz der Arme[n] erlahmen, die heute noch unter Aufgebot aller Kräfte und meistens mit Erfolg bestrebt sind, jenes traurige Schicksal von sich abzuwenden (abgedruckt in Ayass/Tennstedt/Winter 2003: 97).

Diese Aussage illustriert die Befürchtungen in der Industrie zu dieser Zeit, dass durch die Möglichkeit des Leistungsbetrugs Arbeitsanreize und Arbeitsmotivation leiden würden.

Als zusammenfassende Beurteilung lässt sich festhalten, dass bei den Bismarck'schen Reformen für die Industrie Befürchtungen einer Untergrabung von Arbeitsanreizen eine Rolle spielten, dass diese Befürchtungen jedoch nicht zu einer grundsätzlichen Ablehnung dieser Reformen führten, sondern $\mathrm{zu}$ Bestrebungen die Leistungsbestimmungen in diesen Programmen so auszugestalten, dass die Leistungshöhen möglichst gering blieben und an Erwerbstätigkeit geknüpft waren, insbesondere durch die Anbindung von Leistungsansprüchen an Beitragszahlungen der Arbeitgeber und Arbeitnehmer. 
4 Die Einführung der staatlichen Arbeitslosenversicherung

Anders als die anderen klassischen Zweige der Sozialversicherung, stand die Einführung einer Arbeitslosenversicherung nicht auf der Agenda der Bismarck'schen Sozialreformen, sondern erfolgte erst 1927. Eine staatliche Arbeitslosenversicherung wurde bis zur Zeit des Ersten Weltkriegs von allen wesentlichen politischen Akteuren abgelehnt, darunter den meisten Parlamentsparteien, Teilen der Gewerkschaften, sowie den Arbeitgeberverbänden. Aus Sicht der Gewerkschaften stellte eine staatliche Arbeitslosenversicherung eine Konkurrenz zu den gewerkschaftseigenen Unterstützungskassen, welche in der Forschung als „Ghent Modell“ bekannt sind, dar. Diese gewerkschaftlichen Kassen erwiesen sich jedoch durch den Anstieg der Arbeitslosigkeit nach dem Krieg nicht mehr finanzierbar und Teile der Gewerkschaften traten nun für eine staatliche Lösung ein (Faust 1987: 261-262).

Die Einführung einer Kriegserwerbslosenfürsorge 1915 war zunächst explizit als auf die Dauer des Krieges begrenzt angelegt, wurde jedoch im November 1918 im Kontext hoher Arbeitslosigkeit auf provisorischer Basis verlängert. Finanziert und verwaltet wurde die Kriegserwerbslosenfürsorge und ihr Nachfolgeprogramm von en Kommunen, zusammen mit staatlichen Subventionen. Leistungen waren bedarfsgeprüft. Dieses Programm wurde durch die Einführung einer staatlichen Arbeitslosenversicherung durch das Gesetz über Arbeitsvermittlung und Arbeitslosenversicherung (AVAVG) vom 16. Juli 1927 abgelöst. Das Gesetz wurde von einer großen Mehrheit im Reichstag unterstützt und mit 355 gegen 47 Stimmen verabschiedet. Es sah die Einrichtung einer neuen Reichsversicherungsanstalt für die Durchführung der Arbeitsvermittlung und der Arbeitslosenversicherung vor, wobei die Leistungen an Arbeitslose auf dem Versicherungsprinzip beruhten und durch Beiträge von Arbeitgebern und Arbeitnehmern finanziert wurden.

Wie wurde die Einführung der Arbeitslosenversicherung von den politischen Repräsentanten der industriellen Arbeitgeber gesehen? Bis 1918 bestand generelle Einigkeit auf Seite der Arbeitgeberverbände, dass eine staatliche Arbeitslosenversicherung abzulehnen sei. Äußerungen von Arbeitgebervertretern in den politischen Debatten zeigen, dass diese Arbeitslosigkeit nicht als strukturelles Phänomen sondern als ein individuelles Verschulden betrachteten, das heißt, das Ergebnis von Faulheit oder mangelnder Qualifikation.

Auf einer Arbeitsnachweis-Konferenz der Vereinigung deutscher Arbeitgeberverbände (VDA) im November 1913 sprachen sich die anwesenden Vertreter einhellig gegen die Einführung einer Arbeitslosenversicherung aus (Zentrales Staatsarchiv, DAF, 2632/220). In einer Denkschrift von März 1918 sprach sich die VDA erneut gegen eine Arbeitslosenversicherung und gegen eine Fortsetzung der Kriegserwerbslosenfürsorge nach 
Kriegsende aus (VDA 1918). Am 24.April 1920 teilte die VDA dem Reichsarbeitsministerium mit, dass ein großer Teil der Mitglieder den Ministerialentwurf für eine Arbeitslosenversicherung ablehne (Zentrales Staatsarchiv, RAM, 4310/470-476). Der Deutsche Industrie-und Handelstag (DIHT) lehnte die Einführung einer Arbeitslosenversicherung ebenso ab (DIHT 1920; siehe auch Zentrales Staatsarchiv, 29. Oktober 1920, RAM, 4311/197-198).

Welche Argumente führten die Arbeitgeberverbände gegen eine staatliche Arbeitslosenversicherung an? Zwei Argumente dominieren in den Veröffentlichungen und Berichten der Arbeitgeberverbände: Kostensteigerungen und das Argument, dass eine Arbeitslosenversicherung Faulheit belohnen würde. Dieses zweite Argument illustriert klar die Befürchtung eines Rückgangs des Arbeitskräfteangebots. Die folgenden Zitate aus Veröffentlichungen von Arbeitgeberverbänden illustrieren diese Überlegungen. Der Vorsitzende des Nürnberg-Fürther Arbeitgeberkartells, Hans-Xaver Zahnbrecher, argumentierte 1914 in einer Veröffentlichung dieses Verbands zur Frage der Arbeitslosenversicherung: "...die Arbeitskraft unseres Volkes würde erschlaffen, und herabgesetzt werden. Der Zwang, sich nach Arbeit umzusehen, würde mehr oder weniger aufhören und die Leistungsfähigkeit aufhören." (Zahnbrecher 1914: 34). Ein Kommentar in der Deutschen Arbeitgeberzeitung, dem Organ der Arbeitgeberverbände, argumentierte 1920 mit folgenden Argumenten gegen eine Arbeitslosenversicherung:

Ein Volk von einer gesunden Willenskraft und vorwärtsstrebender Energie gibt sich doch nicht selbst ein Gesetz über die Versicherung der Arbeitslosigkeit, d.h. der staatlichen Anerkennung eines Krebsschadens, den Stempel der Minderwertigkeit und der Krüppelhaftigkeit....Hier müssen Menschen gewagt werden, damit ein Volk wird. Verkenne man doch ferner nicht, dass ein gesunder gerechter Kampf um das tägliche Brot durchaus vorteilhaft ist. In diesem Kampfe werden die Sinne geschärft und die Kräfte entfesselt. Der Ehrgeiz erwacht und das Streben nach vorwärts. .... Eine staatliche Versicherung der Arbeitslosigkeit würde zu einer Sorglosigkeit in den unteren Volksschichten führen, ja geradezu die Möglichkeit bieten die Faulenzerei zu züchten. Der innere Antrieb, das feine Schamgefühl nicht auf Kosten der Allgemeinheit zu leben, wird erstickt durch eine staatliche Sanktionierung der Arbeitslosigkeit (DAGZ 1920: 1).

Die Arbeitgeberverbände erwarteten also durch eine Arbeitslosenversicherung negative Effekte auf Arbeitsmotivation und Leistungsbereitschaft. Gleichzeitig finden sich in den verfügbaren Quellen keine Hinweise darauf, dass Arbeitgebervertreter mit positiven Effekten einer Versicherung auf Ausbildungsanreize argumentierte hätten (siehe Paster 2012: 108109).

Die Befürchtung der Arbeitgebervebände, dass eine Arbeitslosenversicherung die 
Arbeitsanreize reduzieren würde kam auch im Beharren auf strenge Zumutbarkeitsregeln für Leistungsempfänger zum Ausdruck. Arbeitslose sollten aus Sicht der Verbände dazu verpflichtet sein auch Stellen anzunehmen die nicht ihrer Ausbildung entsprechen. Die Regierungsvorlage von 1925 sah vor, dass ein Leistungsempfänger eine Stelle annehmen müsse, welche „ihm unter billiger Berücksichtigung seiner Ausbildung und seiner bisherigen Tätigkeit zugemutet werden kann“. Die VDA lehnte diese Bestimmung ab mit der Begründung, dass diese keine „Aussicht auf Zuverlässigkeit und Einheitlichkeit“ habe. In einer Stellungnahme der VDA heißt es dazu ,[b]ei der Zumutbarkeit sollte keine Rücksicht auf die berufliche Vorbildung...genommen werden“ (VDA 1926: 36). Auch der Deutsche Industrie-und Handelstag und der Reichsverband Deutscher Industrie (RDI) traten für strenge Zumutbarkeitsregeln ein (Erklärung des DIHT vom 29. Oktober 1920, Zentrales Staatsarchiv, RAM, 4311/197-198; Brief des RDI vom 18. Dezember 1920 an das Reichsarbeitsministerium, Zentrales Staatsarchiv, RAM, 4311/202-3). Eine Untersuchung der Haltungen deutscher Arbeitgeberverbände zur Arbeitslosenversicherung nach 1945 von Magnus Brosig zeigte, dass auch nach 1945 die Arbeitgeberverbände für strenge Zumutbarkeitsregeln und gegen einen starken Qualifikationsschutz in der Arbeitslosenversicherung eintraten (Brosig 2011).

Trotz der Bedenken gab die VDA ihren Widerstand gegen die Einführung der Arbeitslosenversicherung auf und entschloss sich 1925 zur Kooperation. In einer Sitzung des Sozialpolitischen Ausschusses der VDA am 27. Januar 1925 beschlossen dessen Mitglieder bei der Ausarbeitung des Regierungsentwurfs zu kooperieren, da eine Fortsetzung des Widerstands „keine Aussicht auf Erfolg“ hätte und man durch Kooperation die Berücksichtigung der Interessen der Arbeitgeber in der Ausarbeitung des Entwurfs sicherstellen wollte, beispielsweise im Bezug auf die Begrenzung der Kostensteigerungen durch strenge Anspruchsvoraussetzungen und die Sicherstellung des Einflusses der Verbände in der Verwaltung der neu zu gründenden Versicherungsanstalt (Führer 1990: 218).

Ein Vertreter des Verbands Berliner Metallindustrieller, Stefan Oppenheimer, erläuterte die Beweggründe für diesen Positionswechsel auf einer Tagung der Deutschen Gesellschaft zur Bekämpfung der Arbeitslosigkeit am 20. Februar 1925 wie folgt:

$\mathrm{Da}$ ist es für die Arbeitgeberseite vollkommen klar, daß es, nach den Anträgen, die von allen politischen Parteien gestellt sind, ganz nutzlos wäre, sich auf den Standpunkt zu stellen: wir wollen keine Arbeitslosenversicherung haben! Deshalb ist es der Standpunkt der Arbeitgeber, lediglich zu sagen: die Arbeitslosenversicherung muß sich in dem Rahmen des Erträglichen halten, und unsere Pflicht ist es, auf die Gefahren hinzuweisen, die der Wirtschaft von der Arbeitslosenversicherung drohen können (in Jastrow/Erdmann/Spliedt 1925: 113). 
Der Positionswechsel der Arbeitgeberverbände erscheint daher strategisch motiviert gewesen $\mathrm{zu}$ sein, und ist nicht auf eine geänderte Einschätzung der Auswirkungen der Versicherung auf Arbeitsanreize zurückzuführen (eine ähnlich Einschätzung vertritt: Lewek 1992: 231).

Zusammenfassend lässt sich sagen, dass Befürchtungen der Arbeitgeberverbände einer Erosion von Arbeitsanreizen durch Sozialleistungen bei der Einführung der Arbeitslosenversicherung eine wesentlich größere Rolle spielten als bei der Einführung der anderen Zweige der Sozialversicherung. Dies ist offenbar dadurch verursacht, dass die Arbeitslosenversicherung arbeitsfähigen Personen zugute kommt, während bei der Krankenund Unfallversicherung dies nicht der Fall ist, und bei den Altersrenten eine Erwerbserwartung klarerweise nicht besteht. Dass die Arbeitgeberverbände die Einführung einer staatlichen Arbeitslosenversicherung ungleich stärker ablehnten als die Einführung der anderen Zweige der Sozialversicherung, kann daher durch die stärkere Bedeutung dieser Versicherung für den Erhalt von Arbeitsanreizen erklärt werden.

\section{Der Ausbau der Sozialpolitik nach 1945}

Nach der Debatte um die Einführung einer Einheitsversicherung 1947/1948, verschob sich der Fokus sozialpolitischer Debatten weg von Fragen des institutionellen Designs hin zur Frage der Verbesserung von Leistungen, wobei die Rentenreform von 1957 die wichtigste Einzelreform darstellte. In institutioneller Hinsicht setzten die Reformen dieser Periode das Bismarcksche Sozialmodell; mit Beitragsfinanzierung, einkommensbezogenen Leistungen, und korporatistischer Selbstverwaltung; fort.

Die Positionen der BDA in dieser Zeit unterscheiden sich deutlich von denen der Vorgängerorganisation in der Weimarer Republik, der VDA. Die Grundsatzkritik an arbeitsmarktnahen Sozialprogrammen wurde ersetzt durch ein Bestreben zur arbeitsanreizkompatiblen Ausgestaltung von Sozialprogrammen. Die Veröffentlichungen der 1949 als Nachfolgeorganisation der VDA gegründeten Bundesvereinigung Deutscher Arbeitgeberverbände (BDA) drücken ein grundsätzliches Bekenntnis zu den Prinzipien des Bismarck'schen Sozialstaates aus: einkommensbezogene Leistungen, Beitragsfinanzierung, korporatistische Selbstverwaltung, und Beibehaltung separater Versicherungszweige statt Einheitsversicherung. Betont wurde jedoch von der BDA weiterhin die Notwendigkeit der Aufrechterhaltung von Arbeitsanreizen. Der wesentliche Unterschied zur Weimarer Republik in den sozialpolitischen Positionen des jeweiligen Spitzenverbands besteht darin, dass diese nun nicht mehr staatliche Sozialpolitik an sich als Gefahr für den Erhalt von Arbeitsanreizen sahen, sondern lediglich deren spezifische Ausgestaltung. 
In den Veröffentlichungen der BDA aus dieser Zeit kommt zum Ausdruck, dass sie im bismarckschen Sozialversicherungsprinzip eine Äquivalenz zwischen Erwerbstätigkeit und Leistungsansprüchen gewahrt sah, und daher nun offenbar, im Unterschied zur VDA in der Weimarer Republik, keinen grundsätzlichen Widerspruch zwischen sozialer Absicherung und Erhalt von Arbeitsanreizen sah, zumindest nicht so lange dieses Äquivalenzprinzip gewahrt sei.

In einem 1953 veröffentlichen Grundsatzprogramm der BDA mit dem Titel Gedanken zur sozialen Ordnung kommt diese programmatische Neuorientierung so zum Ausdruck:

Das soziale Gewissen und die Staatsraison fordern, daß die Gemeinschaft eintritt, um denjenigen Mitbürgern die elementaren Voraussetzungen ihrer Existenz zu sichern, die wegen Krankheit, Alter, Invalidität oder sonstiger unabwendbarer Schicksale sich nicht mehr selbst erhalten können....Die Grenzen liegen dort, wo Art und Maß der öffentlichen Betreuung die Verantwortlichkeit des Staatsbürgers im Daseinskampf für sich und seine Familie zu lähmen droht. Die öffentliche Hilfe muß daher subsidiär bleiben. Auch kann sie nicht zu einer gleichförmigen Schicksalsversicherung ausgebaut werden (BDA 1953: 6).

Bemerkenswert ist, dass in der Aufzählung der Risiken Arbeitslosigkeit nicht erwähnt wird, also mutmaßlich von der BDA nicht zu den „unabwendbaren Schicksalen“ gezählt wurde. Als abzusichernde Risiken führt das Grundsatzprogramm explizit lediglich die arbeitsmarktfernen Risiken Krankheit, Alter und Invalidität an. Der Verweis auf die Notwendigkeit von Eigenverantwortlichkeit deutet auf die als für Arbeitsanreize problematisch betrachteten Formen von Sozialpolitik hin.

Explizit verteidigt die BDA in dieser Zeit, und auch bis heute, das Versicherungsprinzip gegenüber dem Fürsorgeprinzip. Diese Positionierung kommt erstmals in einer Stellungnahme der BDA im Kontext der Debatte um die Einheitsversicherung 1949 zum Ausdruck. Darin heißt es:

Der Hauptausschuß der Sozialversicherung der Bundesvereinigung der Deutschen Arbeitgeberverbände vertritt grundsätzlich den Standpunkt, daß das Versicherungsprinzip in der deutschen Sozialversicherung aufrechterhalten werden muß und nicht durch das Prinzip einer Volksfürsorge ersetzt oder verwässert werden darf. ...Die Arbeitgeberschaft bejaht alle erforderlichen Maßnahmen, die zur Wiederherstellung der Leistungsfähigkeit der Sozialversicherung und zu ihrer den Zeitverhältnissen entsprechenden Förderung dienen. (Erdmann 1966: 236).

Es erscheint plausibel, dass die Arbeitsanreizkompatibilität ein wichtiger Grund für die Befürwortung des Versicherungsprinzips durch die BDA war, wenn gleich dieser Zusammenhang in den Publikationen nicht explizit hergestellt wird. Das im Kaiserreich und in der Weimarer Republik häufig von den Spitzenverbänden verwendete Argument, dass 
arbeitsmarktnahe Sozialproramme Arbeitsanreize reduzieren, ist jedenfalls in den Publikationen der Nachkriegszeit nicht ehr zu finden.

6. Die Politik der Frühverrentung in den 1970ern und 1980ern

In der Phase der Restrukturierungen und der Überbeschäftigung in der Industrie ab den 1970ern trat auf der Arbeitgeberseite der Erhalt von Arbeitsanreizen in den Hintergrund. Stattdessen entwickelten einige Unternehmen, primär Großunternehmen in der Industrie, eine Interessensdeutung, welche negative Arbeitsanreize durch Frühverrentung als hilfreich für konfliktarmen Personalabbau interpretierte (Ebbinghaus 2006: 43-45; Jacobs/Kohli/Rein 1991: 200-203; Kohli/Rein 1991: 7-9).

Die BDA vertrat zeitweilig, besonders in den 1980ern, diese Interessensdeutung, wurde jedoch ab den 1990ern kritischer gegenüber staatlichen Maßnahmen zur Erleichterung des vorzeitigen Arbeitsmarktausstiegs (vgl. Mares 2004b). 1985 begrüßte die BDA laut ihrem Jahresbericht beispielsweise die Ausweitung der Arbeitslosenversicherung für ältere Arbeitnehmer auf 24 Monate (BDA 1985: 49). Im Jahresbericht der BDA für 1984 heißt es zum Vorruhestandsgesetz:

Nach Auffassung der Bundesvereinigung bietet die dem Vorruhestand zugrundeliegende Konzeption die Möglichkeit, um das aktuelle, vor allem durch die demographische Entwicklung vorübergehend verursachte Ungleichgewicht auf dem Arbeitsmarkt zu mildern und insbesondere die beruflichen Chancen der in Beschäftigung drängenden geburtenstarken Jahrgänge zu verbessern (BDA 1984: 56).

Gleichzeitig betonte die BDA jedoch zu diesem Zeitpunkt bereits, dass es sich bei der Frühverrentung aus Kostengründen nur um eine Übergangslösung handeln könne. Der Geschäftsführer der BDA, Ernst-Gerhard Erdmann, argumentierte daher 1984 auf einer Podiumsdiskussion mit dem Titel „Probleme eines vorgezogenen Ruhestandes“:

Wenn die Arbeitgeber gleichwohl der Konzeption eines Vorruhestands aufgeschlossen gegenüberstehen, dann nur unter dem Aspekt, daß in einer zeitlich begrenzten Ausnahmesituation besondere zusätzliche Hilfen erwogen werden müssen. Es gelte, die aktuellen, vor allem durch die demographische Entwicklung vorübergehend verursachten Spannungen auf dem Arbeitsmarkt zu mildern und insbesondere die beruflichen Chancen der in der Beschäftigung drängenden geburtenstarken Jahrgänge zu verbessern (Ebert 1984: 1112).

Diese Aussagen illustrieren eine grundsätzliche Akzeptanz einer Reduktion des Arbeitskräfteangebots durch die BDA in dieser Phase, wenn auch nur als vorübergehende Lösung. 
Ab den späten 1980ern wird die Haltung der BDA zur sozialpolitischen Ermöglichung von Frühverrentung kritischer. Die BDA betrachtet solche Maßnahmen nun als zu teuer. Im Jahresbericht von 1989 argumentiert die BDA beispielsweise, dass das Vorruhestandsgesetz (VRG)

... im Großen und Ganzen nicht den hohen Erwartungen entsprochen [hat]. Immerhin wurde ein kleiner Beitrag zur Lösung des Arbeitslosenproblems erbracht, so dass das Gesetz insgesamt nicht als Misserfolg bewertet werden kann. Der Lösungsansatz des VRG war jedoch teuer. (BDA 1989: 60)

Die Reduktion des Arbeitskräfteangebots durch Frühverrentung betrachtete die BDA zu diesem Zeitpunkt vor allem aus Kostengründen als problematisch, die Reduktion des Arbeitskräfteangebots als Ziel stellt die BDA zu diesem Zeitpunkt jedoch nicht in Frage.

Ab Mitte der 1990er misst die BDA der Erhöhung des Arbeitskräfteangebots wieder größere Bedeutung zu und wendet sich nun explizit gegen eine Politik der Frühverrentung. Diese geänderte Haltung illustriert die folgende Aussage des BDA-Präsidenten Dieter Hundt von 2008:

Wir können nicht länger gesunde Mitarbeiter im besten Alter aus dem Job drängen. Angesichts des Fachkräftemangels und der sinkenden Zahl von Schulabgängern benötigen die Betriebe sowohl Junge als auch Ältere.

Jeder Euro, der für unsinnige Frühverrentungen ausgegeben wird, verringert den Spielraum für Lohnerhöhungen und Beitragssenkungen in der Sozialversicherung. Angesichts der demografischen Entwicklung und der hohen Sozialabgabenlast geht an einer Verlängerung der Lebensarbeitszeit kein Weg vorbei. Ich fordere die Koalition auf, den eingeschlagenen Weg zur Verlängerung der Lebensarbeitszeit konsequent fortzusetzen (BDA 2008).

Die BDA führte also in den 1990ern eine Kehrtwende in ihrer Haltung zur Frühverrentung durch, motiviert durch eine geänderte Analyse der Arbeitskräftenachfrage.

Diese Neupositionierung kam auch 2014 bei der Einführung einer abschlagsfreien Rente mit 63 bei langen Versicherungszeiten zum Ausdruck. Diese Reform kritisierte die BDA mit Verweis auf negative Effekte auf das Arbeitskräfteangebot scharf (BDA 2014: 912). Die BDA erneuerte diese Kritik seither mehrmals in Presseerklärungen (Die Welt 2019; Finanznachrichten 2019; DPA 2017). Die zugleich mit dem selben Rentenpaket beschlossenen Verbesserungen bei der Erwerbsminderungsrente, also der Rentenansprüche für Personen die nicht bis zum regulären Antrittsalter arbeiten können, unterstützte die BDA jedoch explizit (BDA 2014: 1, 12). Dies ist ein weiterer Hinweis darauf, dass die BDA Reformvorschläge nun in dieser Periode nicht ausschließlich im Hinblick auf Kosteneffekte, sondern auch auf im Hinblick auf Arbeitsanreizeffekte beurteilt. 
Zusammenfassend zeigt die diachrone Analyse dieser Positionsänderungen der BDA in den letzten fünf Jahrzehnten, dass die BDA die arbeitsanreizreduzierenden Effekte von Sozialpolitik nicht immer als nachteilig wahrnahm, sondern ihre Bewertung von der Situation am Arbeitsmarkt abhängig machte. In den Phasen der Überbeschäftigung, besonders in den 1980ern, konstruierte die BDA ein genuines Arbeitgeberinteresse an Sozialpolitik als Instrument zur Ermöglichung eines konfliktarmen Personalabbaus, wenngleich diese Interessensdeutung eine vorübergehende war. Zusätzlich zur geänderten Arbeitsmarktlage spielten offenbar die höher als erwarteten Kosteneffekte der Frühverrentung eine wichtige Rolle bei der Haltungsänderung der BDA.

\section{Die Arbeitsmarktreformen in den 1990ern und 2000ern}

Das neu erwachte Interesse der Arbeitgeber an einer Erhöhung des Arbeitskräfteangebots ab den 1990ern beinhaltete auch neue Forderungen nach Stärkung von Arbeitsanreizen durch Änderungen in der Sozialpolitik, konkret durch Aktivierungsmaßnahmen und strengeren Leistungsvoraussetzungen. Betont werden von der BDA jedoch auch Auswirkungen wachsender Sozialausgaben auf die Lohnkosten.

In den Publikationen der BDA spiegelt sich dieser Kurswechsel weg von einer politisch gestalteten Reduktion des Arbeitskräfteangebots hin zu einer politisch gestalteten Ausweitung erstmals in einem Grundsatzprogramm von 1994 wieder, welches den Titel Sozialstaat vor dem Umbau. Leistungsfähigkeit und Finanzierbarkeit sichern trägt (BDA 1994). Dieses Dokument unterscheidet sich von den Publikationen der BDA in den 1980ern durch eine stärkere Betonung der Notwendigkeit von Kostensenkungen in der Sozialpolitik, sowie der Stärkung von Arbeitsanreizen. Die BDA begründet die Notwendigkeit zur Stärkung von Arbeitsanreizen in dieser Zeit vorrangig durch das Ziel die hohen Sozialausgaben zu senken. Ähnliche Positionen enthalten auch die sozialpolitischen Grundsatzdokumente der BDA in den folgenden Jahren (z.B. BDA 1998, 2002) und wurden von arbeitgebernahen Think Tank Initiative Neue Soziale Marktwirtschaft vertreten (siehe Kinderman 2016: 595596, 2005). Im Einklang mit dem Ziel, die Sozialpolitik stärker auf die Förderung von Arbeitsanreizen auszurichten, befürwortete die BDA grundsätzlich die Hartz IV und Agenda 2010 Reformen, betrachtete diese jedoch als nicht weitreichend genug.

Der Wechsel von einer „Stillegungspolitik“, welche das sozialverträgliche Ausscheiden von Arbeitslosen aus dem Arbeitsmarkt ermöglichte, hin zu einer Förderung von Arbeitsanreizen durch Aktivierung und restriktiveren Leistungsvoraussetzungen war begleitet von internen Konflikten bei den Arbeitgeberverbänden. Kleinere Unternehmen vor allem 
sahen die staatliche Subventionierung von Frühverrentung schon früh kritisch, da diese Instrumente primär von Großunternehmen genutzt wurden (Hassel/Schiller 2010: 115-117). Gleichzeitig waren die Verbände mit Verbandsaustritten kleinerer Unternehmen konfrontiert (Silvia/Schroeder 2007). Die Rückkehr zur Förderung von Arbeitsanreizen hingegen beruhte auf einem breiteren Konsens innerhalb der Arbeitgeberverbände. Aus Sicht der BDA spricht für eine Stärkung der Arbeitsanreize in der Sozialpolitik nicht nur die Vergrößerung des Arbeitskräfteangebots, sondern im Kontext hoher Sozialausgaben auch die Reduzierung der Abgabenbelastung durch eine reduzierte Zahl an Leistungsempfängern. Die Refokusierung ihrer sozialpolitischen Forderungen auf Aktivierung und Arbeitsanreize ermöglichte es der BDA beide Ziele, Ausweitung des Arbeitskräfteangebots und Senkung der Sozialausgaben, gleichzeitig zu fördern.

\section{Schlussfolgerungen}

Dieser Beitrag hat gezeigt, dass die Gestaltung von Arbeitsanreize eine wesentlicher Faktor in der Beurteilung sozialpolitischer Reformpläne durch die Spitzenverbände der Arbeitgeber ist. Die deutschen Arbeitgeberverbände beurteilen staatliche Sozialpolitik nicht nur im Hinblick auf ihre Kosten für ihre Mitglieder, sondern wesentlich auch im Hinblick auf ihre Auswirkungen auf Arbeitsanreize und das Arbeitskräfteangebot. Die Bedeutung welche die Verbände Arbeitsanreiten beimaßen und wie sie diese bewerteten variierte jedoch abhängig von Arbeitsmarktlage und der Arbeitsmarktnähe des jeweiligen Programs. Drei Ergebnisse sollten abschließend als Forschungsresultate hervorgehoben werden.

Der Beitrag konnte erstens zeigen, dass die Haltungen der Spitzenverbände der Arbeitgeber in Deutschland zur Einführung der wesentlichen Zweige der Sozialversicherung stark mit Befürchtungen einer Schwächung der Arbeitsanreize korrelierten. Von allen Zweigen, lehnten die Spitzenverbände die Einführung der Arbeitslosenversicherung am schärfsten ab. Auf Basis historischer Quellen konnte der Beitrag außerdem zeigen, dass diese besonders starke Ablehnung der Arbeitslosenversicherung auf Befürchtungen von negativen Arbeitsanreizen zurückzuführen ist. Eine deutliche schwächere Rolle spielten Befürchtungen negativer Arbeitsanreize bei der Einführung der Alters- und Invaliditätsrenten, wo der Centralverband solche Anreize durch Möglichkeiten zum Leistungsbetrug vermutete. Bei der Einführung der Unfall- und der Krankenversicherung fand die Untersuchung jedoch keine Hinweise, dass die Verbände negative Arbeitsanreize befürchteten. Die Arbeitgeberverbände begrüßten grundsätzlich die Einführung dieser Programme. 
Der Beitrag konnte, zweitens, durch einen diachronen Vergleich von Reformepisoden über etwa 130 Jahre zeigen, wie sich die Bewertung der Bedeutung von Arbeitsanreizen im Zeitverlauf veränderte (siehe Tabelle 1). Im Kaiserreich und in der Weimarer Republik lehnten die Verbände jene Sozialprogramme, denen sie negative Arbeitsanreize zuschrieben ab. Diese Grundsatzkritik an Sozialpolitik verschwand nach 1945. Die BDA in den Nachkriegsjahrzehnten konzentrierte sich, im Unterschied zu ihren Vorgängerorganisationen, auf die Ausgestaltung von Sozialpolitik im Einklang mit dem Ziel des Erhalts von Arbeitsanreizen, und nicht auf Grundsatzkritik an Sozialpolitik.

Später, in der Zeit des Strukturwandels in der Industrie in den 1970ern und 1980ern, begrüßten die BDA die Schaffung negativer Arbeitsanreize durch Sozialpolitik. Seit den 1990er fordert die BDA jedoch, vor dem Hintergrund gestiegener Sozialausgaben und Arbeitskräftemangel, wieder eine Stärkung von Arbeitsanreizen in der Sozialpolitik. Zusammenfassend lässt sich also feststellen, dass die Beurteilung negativer Arbeitsanreize durch die Verbände zwar ursprünglich eine negative war, jedoch im Kontext von Überbeschäftigung eine positive wurde. Die Beurteilung der Bedeutung von Arbeitsanreizen durch die untersuchten Spitzenverbände variiert also nicht nur in ihrer Intensität, sondern auch in ihrer Richtung.

Ein drittes Ergebnis des Beitrags ist, dass die deutschen Arbeitgeberverbände konsistent, das heißt, über den gesamten Zeitraum von den 1880ern bis heute, das Versicherungsprinzip, charakterisiert durch einkommensbezogene Leistungen und Beitragsfinanzierung, gegenüber dem Sozialhilfeprinzip, charakterisiert durch bedarfsgeprüfte Leistungen und Steuerfinanzierung, bevorzugten. Diese Position kommt in allen programmatischen Dokumenten der BDA und ihrer Vorgängerorganisationen zum Ausdruck. In der Debatte um die Einführung einer Arbeitslosenversicherung in den 1920ern war ein Motiv für die Kehrtwende der Arbeitgeberverbände von Gegnern zu Befürwortern des Versicherungsmodells, zusätzlich zu strategischen Überlegungen, das Ziel die bedarfsgeprüfte Erwerbslosenfürsorge zu beseitigen. Wenngleich die Gründe für diese Positionierung vielfältig sind, deuten die Stellungnahmen der Verbände darauf hin, dass der Schutz von Arbeitsanreizen ein relevantes Motiv darstellt. 


\section{Literaturverzeichnis}

Ayass, Wolfgang; Tennstedt, Florian; Winter, Heidi (Hg.) (2003): Von der Kaiserlichen Sozialbotschaft bis zu den Februarerlassen Wilhelm II. (1881-1890) Band 1. Grundfragen der Sozialpolitik: die Diskussion der Arbeiterfrage auf Regierungsseite und in der Öffentlichkeit Quellensammlung zur Geschichte der Deutschen Sozialpolitik 1867 bis 1914. Darmstadt: Wissenschaftiche Buchgesellschaft.

Baare, Louis, 1880: Protokoll der Konferenz in Bochum am 2. und 3. November 1880. Potsdam: Bundesarchiv Potsdam. Bd. 15.01.

BDA, Bundesvereinigung der Deutschen Arbeitgeberverbände (1953): Gedanken zur sozialen Ordnung. Der Öffentlichkeit übergeben von der Bundesvereinigung der Deutschen Arbeitgeberverbände. Köln, Heiderdruck Bergisch Gladbach.

BDA, Bundesvereinigung der Deutschen Arbeitgeberverbände (1984): Jahresbericht der Bundesvereinigung der Deutschen Arbeitgeberverbände. Köln: BDA.

BDA, Bundesvereinigung der Deutschen Arbeitgeberverbände (1985): Jahresbericht der Bundesvereinigung der Deutschen Arbeitgeberverbände. Köln: BDA.

BDA, Bundesvereinigung der Deutschen Arbeitgeberverbände (1989): Jahresbericht der Bundesvereinigung der Deutschen Arbeitgeberverbände. Köln: BDA.

BDA, Bundesvereinigung der Deutschen Arbeitgeberverbände (1994): Sozialstaat vor dem Umbau. Leistungsfähigkeit und Finanzierbarkeit sichern. Bundesvereinigung der deutschen Arbeitgeberverbände. Köln: BDA.

BDA, Bundesvereinigung der Deutschen Arbeitgeberverbände (1998): Sozialpolitik für mehr Wettbewerbsfähigkeit und Beschäftigung. Ordnungspolitische Grundsätze der Bundesvereinigung der Deutschen Arbeitsgeberverbände. Köln: BDA.

BDA, Bundesvereinigung der Deutschen Arbeitgeberverbände (2002): Neuaufbruch in der sozialen Sicherung. Köln: BDA.

BDA, Bundesvereinigung der Deutschen Arbeitgeberverbände (2008): Arbeitgeberpräsident Dr. Dieter Hundt: Eine Rückkehr zur Frühverrentung darf es nicht geben, PresseInformation Nr. 050/2008. Berlin: BDA, Download unter http://www.bdaonline.de/www/arbeitgeber.nsf/res/PI05008.pdf/\$file/PI05008.pdf (Zugriff am 27. Juli 2019).

BDA, Bundesvereinigung der Deutschen Arbeitgeberverbände (2014): Rentenpaket: Annahme verweigern: Stellungnahme zum Entwurf eines „Gesetzes über Leistungsverbesserungen in der gesetzlichen Rentenversicherung" (RVLeistungsverbesserungsgesetz). Berlin: BDA.

Breger, Monika (1982): Die Haltung der industriellen Unternehmer zur staatlichen Sozialpolitik in den Jahren 1878-1891. Frankfurt am Main: Haag + Herchen.

Breger, Monika (1994): „Der Anteil der deutschen Großindustriellen an der Konzeptualisierung der Bismarckschen Sozialgesetzgebung", in: Lothar Machtan (Hg.), Bismarcks Sozialstaat: Beiträge zur Geschichte der Sozialpolitik und zur sozialpolitischen Geschichtsschreibung.. Frankfurt am Main u.a.: Campus, 25-60.

Bridgen, Paul (2016): „UK employers and the development of state protection for unemployment, sickness and old age, 1900-1990", in: Dennie Oude Nijhuis (Hg.), Business Interests and the Development of the Modern Welfare State. Routledge Studies in the Political Economy of Welfare, Abingdon: Routledge.

Brosig, Magnus (2011): „Sozialpolitik als Hilfe für die Wirtschaft? Deutsche Arbeitgeber und die Systeme der Arbeitslosenversicherung", Zeitschrift für Sozialreform 57: 313-337.

Bueck, Henry Axel (1905a): Der Centralverband Deutscher Industrieller, 1876-1901. Bd. 2. Berlin: J. Guttentag.

Bueck, Henry Axel (1905b): Die Organisation der Arbeitgeber. Berlin: J. Guttentag. 
Bührer, Werner (2010): „Die Europapolitik der deutschen Wirtschafts- und Arbeitgeberverbände", in: Wolfgang Schroeder; Bernhard Wessels (Hg.), Handbuch Arbeitgeber- und Wirtschaftsverbände in Deutschland. 1. Aufl.. Wiesbaden: VS, 457482.

Busemeyer, Marius R. (2011): „Varieties of Cross-Class Coalitions in the Politics of Dualization: Insights from the Case of Vocational Training in Germany", Köln: MaxPlanck-Institut für Gesellschaftsforschung, MPIfG Discussion Papers 11/13.

Callaghan, Helen (2011): „Constrain-Thy-Neighbor Effects as a Determinant of Transnational Interest Group Cohesion", Comparative Political Studies 44: 910-931.

DAGZ, Deutsche Arbeitgeberzeitung (1920): „Die moralischen Gefahren der reichsgesetzlichen Regelung der Arbeitslosen-Versicherung", in Die Deutsche Arbeitgeber-Zeitung. Zentralblatt deutscher Arbeitgeberverbände, Nr. 44 (31. Oktober), 1-2.

DIHT, Deutscher Industrie- und Handelstag (1920): „Arbeitslosenversicherung", in: Handel und Gewerbe. Zeitschrift für die zur Vertretung von Handel und Gewerbe gesetzlich berufenen Körperschaften. Im Auftrag des Deutschen Industrie- und Handelstags., Nr. 6: 56-57.

Die Welt (2019): „BDA-Chef Kampeter „Rente mit 63 ist eine schwere Hypothek", in: Die Welt, $24 . \quad$ April, Downolad unter https://www.welt.de/wirtschaft/article192368261/BDA-Chef-Kampeter-Rente-mit-63ist-eine-schwere-Hypothek.html (Zugriff am 27. Juli 2019).

DPA, Deutsche Presseagentur (2017): „Run auf Rente mit 63: Rente mit 63 verschärft den Fachkräftemangel", in: Wirtschaftswoche, 21. April, Download unter https://www.wiwo.de/erfolg/trends/run-auf-rente-mit-63-rente-mit-63-verschaerft-denfachkraeftemangel/19699590.html (Zugriff am 27. Juli 2019).

Ebbinghaus, Bernhard (2001): „When labour and capital collude: the political economy of early retirement in Europe, Japan and the USA", in: Bernhard Ebbinghaus; Philip Manow (Hg.), Comparing Welfare Capitalism. Social policy and political economy in Europe, Japan and the USA. Routledge/EUI Studies in the Political Economy of Welfare, London u.a.: Routledge.

Ebbinghaus, Bernhard (2006): Reforming Early Retirement in Europe, Japan and the USA. Oxford: Oxford Univ. Press.

Ebbinghaus, Bernhard (2010): „Unions and Employers", in: Francis G. Castles; Jane Lewis; Stephan Leibfried; Herbert Obinger; Christoph Pierson (Hg.), The Oxford Handbook of the Welfare State. Oxford: Oxford Univ. Press, 196-210.

Ebert, Reinhard (1984): „Probleme eines vorgezogenen Ruhestands", Der Arbeitgeber, 36: 11-12.

Eichhorst, Werner; Weishaupt, Timo J. (2013): „Mit Sozialpartnerschaft durch die Krise? Die Rolle von Arbeitgebern und Gewerkschaften in Deutschland, Österreich und der Schweiz", Zeitschrift für Sozialreform 59: 313-335.

Emmenegger, Patrick; Marx, Paul (2011): „Business and the development of job security regulations: the case of Germany", Socio-Economic Review 9: 729-756.

Erdmann, Gerhard (1966): Die deutschen Arbeitgeberverbände im sozialgeschichtlichen Wandel der Zeit. Neuwied: Luchterhand.

Esping-Andersen, Gøsta (1990): The Three Worlds of Welfare Capitalism. Princeton: Princeton Univ. Press.

Estévez-Abe, Margarita; Iversen, Torben; Soskice, David (2001): „Social Protection and the Formation of Skills: A Reinterpretation of the Welfare State", in: Peter A. Hall; David Soskice (Hg.), Varieties of Capitalism. The Institutional Foundations of Comparative Advantage. Oxford: Oxford Univ. Press, 145-183. 
Faust, Anselm (1987): „Von der Fürsorge zur Arbeitsmarktpolitik: Die Errichtung der Arbeitslosenversicherung", in: Werner Abelshauser (Hg.), Die Weimarer Republik als Wohlfahrtsstaat. Stuttgart: Steiner, 260-279.

Finanznachrichten (2019): Arbeitgeber: Die Rente mit 63 verschärft den Fachkräftemangel. Download unter https://www.finanznachrichten.de/nachrichten-2019-04/46538095arbeitgeber-die-rente-mit-63-verschaerft-den-fachkraeftemangel-259.htm (Zugriff am 27. Juli 2019).

Francke, L. (1881): „Die Stimmen der deutschen Handels- und Gewerbekammern über das Haftpflicht-gesetz vom 7. Juni 1871 und dem Reichs-UnfallversicherungsGesetzentwurf vom 8. März 1881", Zeitschrift des Königlich-Preussischen Statistischen Bureaus, 21: 397-416.

Führer, Karl Christian (1990): Arbeitslosigkeit und die Entstehung der Arbeitslosenversicherung in Deutschland 1902 - 1927. Berlin: Colloquium.

Hall, Peter A.; Soskice, David (2001): „An Introduction to Varieties of Capitalism", in: Peter A. Hall; David Soskice (Hg.), Varieties of Capitalism. The Institutional Foundations of Comparative Advantage. New York: Oxford Univ. Press, 1-68.

Hassel, Anke; Schiller, Christof (2010): Der Fall Hartz IV: Wie es zur Agenda 2010 kam und wie es weitergeht. Frankfurt: Campus.

Höpner, Martin; Waclawczyk, Maximilian (2012): „Opportunismus oder Ungewissheit? Mitbestimmte Unternehmen zwischen Klassenkampf und Produktionsregime", Köln: Max-Planck-Institut für Gesellschaftsforschung, MPIfG Discussion Paper 12/1.

Iversen, Torben (2005): Capitalism, Democracy, and Welfare. Cambridge Studies in Comparative Politics. Cambridge: Cambridge Univ. Press.

Jacobs, Klaus; Kohli, Martin; Rein, Martin (1991): „Testing the industry-mix hypothesis of early exit", in: Martin Kohli; Martin Rein; Anne-Marie Guillemard; Herman Van Gunsteren (Hg.), Time for retirement. Comparative study of early exit from the labor market. Cambridge: Cambridge Univ. Press, 67-96.

Jastrow, Ignaz; Erdmann, Gerhard; Spliedt, Franz (1925): Das Problem der Arbeitslosenversicherung in Deutschland: Verhandlungen der Deutschen Gesellschaft zur Bekämpfung der Arbeitslosigkeit am 20. Februar 1925 zu Berlin mit Berichten. Schriften der Deutschen Gesellschaft zur Bekämpfung der Arbeitslosigkeit. Berlin: Reimar Hobbing.

Kaelble, Hartmut (1967): Industrielle Interessenpolitik in der Wilhelminischen Gesellschaft. Centralverband Deutscher Industrieller 1895-1914. Berlin: de Gruyter.

Kinderman, Daniel (2005): „Pressure from without, Subversion from within: The TwoPronged German Employer Offensive", Comparative European Politics 3: 432-463.

Kinderman, Daniel (2016): „Challenging varieties of capitalism's account of business interests: Neoliberal think-tanks, discourse as a power resource and employers' quest for liberalization in Germany and Sweden ", Socio-Economic Review 15: 587-613.

Kohli, Martin; Rein, Martin (1991): „The changing balance of work and retirement", in: Martin Kohli; Martin Rein; Anne-Marie Guillemard; Herman Van Gunsteren (Hg.), Time for retirement. Comparative studies of early exit from the labor force. Cambridge: Cambridge Univ. Press, 1-35.

Kohli, Martin; Rein, Martin; Guillemard, Anne-Marie; van Gunsteren, Herman (Hg.) (1991): Time for retirement. Comparative studies of early exit from the labor force. Cambridge Cambridge Univ. Press.

Korpi, Walter (2006): „Power Resources and Employer-Centered Approaches in Explanations of Welfare States and Varieties of Capitalism: Protagonists, Consenters, and Antagonists", World Politics 58: 167-206.

Lewek, Peter (1992): Arbeitslosigkeit und Arbeitslosenversicherung in der Weimarer Republik 1918-1927. Stuttgart: Franz Steiner. 
Mares, Isabela (2003): The Politics of Social Risks. Business and Welfare State Development. Cambridge Studies in Comparative Politics. Cambridge: Cambridge University Press.

Mares, Isabela (2004a): „Economic Insecurity and Social Policy Expansion: Evidence from Interwar Europe", International Organization 58: 745-774.

Mares, Isabela (2004b): Warum die Wirtschaft den Sozialstaat braucht. Ein historischer Ländervergleich. Frankfurter Beiträge zu Wirtschafts- und Sozialwissenschaften, Frankfurt: Campus.

Münnich, Sascha (2010): Interessen und Ideen. Die Entstehung der Arbeitslosenversicherung in Deutschland und den USA. Frankfurt: Campus.

Nützenadel, Alexander (Hg.) (2017): Das Reichsarbeitsministerium im Nationalsozialismus: Verwaltung - Politik - Verbrechen. Göttingen: Wallstein.

Pancaldi, Federico (2011): „Capitalists Against Crisis: Employers and Short-Time Work in Germany and Italy, 20082010", Turin: Collegio Carlo Alberto, Carlo Alberto Notebooks. Nr.222, Download unter http://www.carloalberto.org/assets/workingpapers/no.222.pdf (Zugriff am 27. Juli 2019).

Paster, Thomas (2012): The Role of Business in the Development of the Welfare State and Labor Markets in Germany. Containing Social Reforms Routledge/EUI Studies in the Political Economy of Welfare. London: Routledge.

Paster, Thomas (2013): „Business and Welfare State Development: Why Did Employers Accept Social Reforms?", World Politics 65: 416-451.

Paster, Thomas (2015): „Bringing Power Back In: A Review of the Literature on the Role of Business in Welfare State Politics", Köln: Max-Planck-Institut für Gesellschaftsforschung, MPIfG Discussion Papers 15/03.

Paster, Thomas (2017): „Die Rolle der Arbeitgeber in der Sozialpolitik", in: Wolfgang Schroeder; Bernhard Weßels (Hg.), Handbuch Arbeitgeber- und Wirtschaftsverbände in Deutschland. Wiesbaden: Springer, 487-514.

Paster, Thomas (2019): „Varieties of Capitalism und Sozialpolitik: Thesen und empirische Befunde", in: Herbert Obinger; Manfred G. Schmidt (Hg.), Handbuch Sozialpolitik. Wiesbaden: Springer, 255-273.

Paster, Thomas (2020): „Business Interests and the Development of the German Welfare State", in: Dennie Oude Nijhuis (Hg.), Business Interests and the Development of the Modern Welfare State. Routledge/EUI Studies in the Political Economy of Welfare. Abingdon: Routledge.

Schroeder, Wolfgang (2006): „Selbstverwaltungskorporatismus und neuer Sozialstaat", Zeitschrift für Sozialreform 52: 253-271.

Silvia, Stephen J.; Schroeder, Wolfgang (2007): „Why Are German Employers Associations Declining?: Arguments and Evidence", Comparative Political Studies 40: 1433-1459.

Streeck, Wolfgang (2016): „Von Konflikt ohne Partnerschaft zu Partnerschaft ohne Konflikt: Industrielle Beziehungen in Deutschland", Industrielle Beziehungen 23: 47-60.

Trampusch, Christine (2004): „Vom Klassenkampf zur Riesterrente: Die Mitbestimmung und der Wandel der Interessen von Gewerk-schaften und Arbeitgeberverbänden an der betrieblichen und tariflichen Sozialpolitik", Zeitschrift für Sozialreform 50: 223-254.

Ullmann, Hans-Peter (1979): „Industrielle Interessen und die Entstehung der deutschen Sozialversicherung 1880-1889", Historische Zeitschrift 229: 574 - 610.

VDA, Vereinigung deutscher Arbeitgeberverbände (1918): Forderungen der Übergangszeit und der Friedenswirtschaft (Denkschrift). Berlin: Vereinigung deutscher Arbeitgeberverbände.

VDA, Vereinigung deutscher Arbeitgeberverbände (1926): Stellungnahme zu den Grundfragen der Arbeitslosenversicherung. Berlin: Vereinigung der Deutschen Arbeitgeberverbände. 
Zahnbrecher, Franz X. (1914): Arbeitslosenversicherung und Arbeitgeber. Nürnberg: K. Hofbuchhandlung Schrag. 